\title{
A CRITERION FOR CELLULARITY IN A TOPOLOGICAL 4-MANIFOLD
}

\author{
DUŠAN REPOVŠ
}

\begin{abstract}
We give a necessary and sufficient condition for a compact set in the interior of a topological 4-manifold to be cellular. This generalizes D. R. McMillan's cellularity criterion for higher dimensions to dimension four.
\end{abstract}

Let $X$ be a compact set in the interior of a topological $n$-manifold with (possibly empty) boundary $M$. We say that $X$ is cellular in $M$ if there is a sequence of $n$-cells $C_{i} \subset$ int $M$ such that $X=\bigcap_{i=1}^{\infty} C_{i}$ and $C_{i+1} \subset \operatorname{int} C_{i}$ for every $i$. The concept of cellularity has been one of the most important in modern geometric topology ever since it was introduced by M. Brown [1] in 1960 (e.g., see [5]). In 1964, D. R. McMillan, Jr. [6] proved a beautifully simple criterion for a compact set in the interior of a PL $n$-manifold $(n \geq 5): A U V^{\infty}$-compactum $X$ in the interior of a PL $n$-manifold $(n \geq 5) M$ is cellular in $M$ if and only if it satisfies the following cellularity criterion (CC):

For every neighborhood $U \subset M$ of $X$ there is a neighborhood $V \subset$

$U$ of $X$ such that every loop in $V-X$ is null-homotopic in $U-X$.

(A compact set $X$ in an ANR $Y$ has the $U V^{\infty}$-property [5] if for every neighborhood $U \subset Y$ of $X$ there is a neighborhood $V \subset U$ such that $V$ is contractible in $U$.) By [4], McMillan's result now holds also for TOP $n$-manifolds $(n \geq 5)$. With a special proviso concerning possible fake cubes, McMillan's criterion holds in dimension three as well [6]. Finally, for $n \leq 2$, the Phragmén-Brouwer theorem shows that $X \subset$ int $M^{n}$ is cellular if and only if $X$ has property $u v^{\infty}$ (the homology version of $\left.U V^{\infty}[\mathbf{5}]\right)$ [9]. We extend McMillan's criterion to dimension four:

THEOREM. Suppose that $X$ is a $U V^{\infty}$-compactum in the interior of a topological 4-manifold with boundary $M^{4}$. Then $X$ is cellular in $M^{4}$ if and only if $X$ satisfies the cellularity criterion (CC).

PROOF. It is clear that the condition CC is necessary for cellularity of $X$ in $M^{4}$ [1] so we only prove its sufficiency. Since $X$ is a $U V^{\infty}$-compactum, given a neighborhood $U \subset \operatorname{int} M^{4}$ there is an open set $V \subset U$, containing $X$, and such that $V$ is contractible in $U$. Therefore $V$ is orientable and the classifying map for the topological microbundle on $V$ lifts to $\mathrm{BO}(4)$.

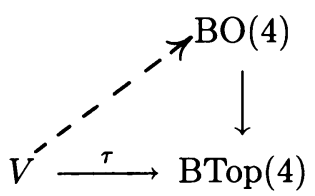

Received by the editors February 11, 1986.

1980 Mathematics Subject Classification. Primary 57N60, 57N13; Secondary 57N25, 57N45.

Key words and phrases. Cellularity criterion, cell-like mapping, 4-manifold.

Research supported in part by the Research Council of Slovenia. 
so $V$ admits a smooth structure [4]. Consider the (noncompact) smooth 4-manifold $N^{4}=V-X$ and let $e$ be its end "near" $X$. The CC implies that $e$ is 1-connected, hence it is topologically collared as $S^{3} \times[0,1)$ by $[3,(1.12)]$. Let $Y^{4}=X+($ the collar) and consider the quotient mapping $q: Y^{4} \rightarrow Y^{4} / X$. Then $q$ is a proper $U V^{\infty}$-mapping between topological 4-manifolds $Y^{4}$ and $Y^{4} / X=B^{4}$ (the 4 -ball) so it can be approximated by homeomorphisms $[7,(2.6 .2)]$. The conclusion now follows by [1] .

REMARK 1 . Note that by $[8,(1.1)], X$ can be expressed as the intersection of a properly nested sequence of smooth 4-balls in $M^{4}$ (if it satisfies CC).

REMARK 2. In [3, (1.11)], M. Freedman formulated a version of the cellularity criterion for topological 4-manifolds which required only that $X$ should have the Čech homology of a point (and satisfy CC). Under the additional assumption that $X$ is simply connected (which was the context of $[3]$ ), this condition suffices and the proof given in [3] is correct. However, this condition is not sufficient if $X$ is not simply connected, as the following example shows: let $M^{4}$ be any nonsimply connected Z-homology 4-sphere, $C^{4} \subset M^{4}$ a nice (tame) 4-ball in $M^{4}$, and define $X=M^{4}-$ int $C^{4}$. Then it is straightforward to check that $\tilde{H}_{*}(X ; \mathbf{Z}) \cong 0$ (use the Mayer-Vietoris sequence for the triple $\left.\left(M^{4}, C^{4}, X\right)\right)$. Also, $X$ satisfies CC: given any neighborhood $U \subset M^{4}$ take $V \subset U$ to be $X$ plus a small collar on $\partial C^{4}$ in $C^{4}$ which lies entirely inside $U$. Then

$$
V-X=\partial X \times[0,1) \simeq S^{3}
$$

so

$$
\pi_{1}(V-X) \rightarrow \pi_{1}(U-X)
$$

is the zero homomorphism. On the other hand $X$ clearly isn't cellular for otherwise $M^{4}=C^{4} \cup_{\partial C} X$ would be the 4-sphere. In fact, $X$ is not even 1- $U V$ because there are nontrivial loops in $M^{4}$. That the $1-U V$ property is crucial follows by [5, (2.3)]: $X$ is $U V^{\infty}$ if and only if it has the Cech cohomology with integer coefficients of the point and the 1- $U V$-property. (With the additional hypothesis that $X$ be $1-U V$, the argument in [3, p. 373] can be used to yield another proof of our theorem.)

REMARK 3. The referee pointed out that the oversight in [3] was also detected by F. D. Ancel, R. D. Edwards, and possibly some others. The correct statement of the cellularity criterion (without proof) will also appear in the forthcoming book by R. J. Daverman [2, Chapter 18].

ACKNOWLEDGMENTS. This note was prepared during my visit to the Mathematical Sciences Research Institute at Berkeley in 1985. I wish to acknowledge R. C. Kirby for the invitation and the National Academy of Sciences, U.S.A., for the financial support.

\section{REFERENCES}

1. M. Brown, A proof of the generalized Schoenfies theorem, Bull. Amer. Math. Soc. 66 (1960), 74-76.

2. R. J. Daverman, Decompositions of manifolds, Academic Press, New York, 1986.

3. M. H. Freedman, The topology of four-dimensional manifolds, J. Differential Geom. 17 (1982), 357-453.

4. R. C. Kirby and L. C. Siebenmann, Essays on topological manifolds, Ann. of Math. Studies, No. 88, Princeton Univ. Press, Princeton, N. J., 1977. 
5. R. C. Lacher, Cell-like mappings and their generalizations, Bull. Amer. Math. Soc. 83 (1977), 495-552.

6. D. R. McMillan, Jr., A criterion for cellularity in a manifold, Ann. of Math. (2) 79 (1964), 327-337.

7. F. S. Quinn, Ends of maps. III: Dimensions 4 and 5, J. Differential Geom. 17 (1982), 503-521.

8. __, Smooth structures on 4-manifolds, Four-Manifold Theory (C. Gordon and R. Kirby, eds.), Contemp. Math., vol. 35, Amer. Math. Soc., Providence, R.I., 1984, pp. 473-479.

9. G. T. Whyburn, Analytic topology, Amer. Math. Soc. Colloq. Publ., vol. 28, Amer. Math. Soc., Providence, R.I., 1942.

DEPARTMENT OF MATHEMATICS, UNIVERSity OF LJUBlJANA, JADRANSKa 19, LJUBLJANA, YUGOSLAVIA 61000

Department of Mathematics, University of Texas at Austin, Austin, Texas 78712 